\title{
Análisis de los Términos Utilizados en la Literatura Científi- ca para Referirse a los Ligamentos Extracapsulares de la Articulación Témporomandibular. Parte II: Ligamentum stylomandibulare y Raphe pterymandibulare
}

\author{
Analysis of Terms Used in Scientific Literature Referred to Extracapsular Ligaments of the \\ Temporomandibular Joint. Part II: Ligamentum stylomandibulare and Raphe pterymandibulare
}

\author{
Ramón Fuentes $^{*, * * ;}$; Nicolás Ottone ${ }^{* *}$; Mario Cantin ${ }^{*, * *, * * * * *}$ \& Cristina Bucchi, ${ }^{* * * *}$
}

FUENTES, R.; OTTONE, N.; CANTIN, M. \& BUCCHI, C. Análisis de los términos utilizados en la literatura científica para referirse a los ligamentos extracapsulares de la articulación témporomandibular. Parte II: Ligamentum stylomandibulare y Raphe pterymandibulare. Int. J. Morphol., 32(4):1289-1295, 2014.

RESUMEN: La Terminología Anatómica Internacional (TAI) es la consecuencia de siglos de trabajo científico tendientes a unificar los términos para denominar a las estructuras anatómicas del cuerpo humano y facilitar la comunicación entre la comunidad científica. Sin embargo, su uso en la literatura no se ha aplicado por completo. El objetivo de esta investigación fue analizar la bibliografía en relación a la correcta implementación de los términos referentes al ligamento estilomandibular y al rafe pterigomandibular. Se realizó una revisión en libros de anatomía y artículos científicos relacionados. Se encontraron diferencias en cuanto a los términos usados para referirse tanto al ligamento estilomandibular y al rafe pterigomandibular, como a las estructuras que sirven de origen e inserción a estos, al compararlos con los términos establecidos por la TAI en latín, inglés y español.

PALABRAS CLAVE: Ligamento estilomandibular; Ligamento pterigomandibular; Rafe pterigomandibular; Terminología Anatómica Internacional.

\section{INTRODUCCIÓN}

La Terminología Anatómica Internacional (TAI) (1998, 2001) proporciona un listado de términos internacionalmente aceptados para la anatomía topográfica, obtenido tras varios siglos de estudios morfológicos. La TAI determina la supresión de los epónimos, la utilización de un solo término para referirse a cada estructura del cuerpo humano y el valor informativo de estos términos. Los esfuerzos por establecer una denominación única han sido numerosos, pero el uso de términos obsoletos sigue siendo una práctica común en el mundo científico.

La articulación temporomandibular (ATM) está dispuesta entre el cóndilo de la mandíbula, el tubérculo articular y la fosa mandibular del hueso temporal (Grau et al., 2005). Además, está compuesta por superficies articulares, el disco articular, sinoviales y ligamentos. El Ligamentum stylomandibulare es uno de los ligamentos de la articulación témporomandibular (ATM), así como el raphe pterymandibulare (TAI, 2001), que es mencionado por varios autores como un ligamento de la ATM (Martínez Ross, 1992; Velayos \& Santana, 2001; Giambartolomei, 2003; Lopes, 2004; Latarjet \& Ruiz Liard, 2005; Rubiano, 2005; Sosa, 2006).

En la literatura científica se observan diferencias en la denominación, clasificación y función del ligamento estilomandibular y el rafe pterigomandibular, y también existen discrepancias con respecto a cuales son y como se denominan las estructuras que sirven de origen e inserción a ellos.

El objetivo de esta investigación fue analizar la literatura científica, libros y artículos, en relación al uso de los

\footnotetext{
* Programa Magister en Odontología, Facultad de Odontología, Universidad de La Frontera, Temuco, Chile.

** Centro de investigación CIMOFIR, Facultad de Odontología, Universidad de La Frontera, Temuco, Chile.

*** Centro de investigación CIMA, Facultad de Odontología, Universidad de La Frontera, Temuco, Chile.

***** Centro de Investigación en Ciencias Biomédicas, Universidad Autónoma de Chile, Temuco, Chile.
} 
FUENTES, R.; OTTONE, N.; CANTIN, M. \& BUCCHI, C. Análisis de los Términos Utilizados en la Literatura Científica para Referirse a los Ligamentos Extracapsulares de la Articulación Témporomandibular Parte II: Ligamentum stylomandibulare y Raphe pterymandibulare. Int. J. Morphol., 32(4):1289-1295, 2014.

términos referentes a la descripción, clasificación, origen, inserción y función del ligamento estilomandibular y del rafe pterigomandibular y contrastar la utilización de estas denominaciones con las establecidas por la Terminología Anatómica Internacional. De esta forma, se complementa el trabajo realizado previamente con respecto al ligamento esfenomandibular.

\section{MATERIAL Y MÉTODO}

Se realizó una revisión narrativa de la literatura. Se analizaron 20 libros de ciencias morfológicas, de idiomas español, portugués e inglés y que trataban sobre la articulación temporomandibular. Se estudió específicamente lo referente al ligamento estilomandibular (Ligamentum stylomandibulare) y al rafe pterigomandibular (Raphe pterymandibulare). Se realizó una búsqueda bibliografía de artículos científicos en PubMed, ScienceDirect y SciELO. Se utilizaron las palabras clave: Ligamento estilomandibular, Ligamento pterigomandibular, Rafe pterigomandibular y Terminología Anatómica Internacional. Se incluyeron estudios observacionales realizados en cráneos humanos y revisiones bibliográficas en español e inglés.

Los términos utilizados en los textos revisados, fueron comparados entre sí y con la Terminología Anatómica Internacional en su versión de latín, inglés y español y se procedió a su análisis. En los casos de libros escritos en inglés y portugués, los términos se tradujeron al español de manera literal.

\section{RESULTADOS}

Denominación. Los términos encontrados en esta revisión para referirse al ligamento estilomandibular y rafe pterigomandibular se encuentran en las Tablas I y II, respectivamente.

\section{Clasificación del ligamento estilomandibular (Ligamentum stylomandibulare).}

Mientras que algunos autores lo clasifican como uno de los dos ligamentos accesorios de la ATM, en conjunto con el ligamento esfenomándibular (Okeson; Wurgaft \& Montenegro; Maciel, Pertes \& Gross; Velayos \& Santana; Alves \& Candido; Isberg; Sicher y Dubrul), otros lo clasifican como uno de los tres ligamentos accesorios (Sosa; Lopes; Giambartolomei), auxiliares (Rubiano; Martinez Ross), asociados (Katchburian \& Arana), de acción indirecta (Giambartolomei) o extrínsecos (Latarjet \& Ruiz; Lopes) de la ATM. Estos tres ligamentos corresponden al ligamento esfenomándibular, estilomándibular y rafe pterigomándibular, según esos autores. Sólo MacKinnon \& Morris (1993) lo menciona como ligamento extracapsular, que es la clasificación utilizada por TAI (2001).

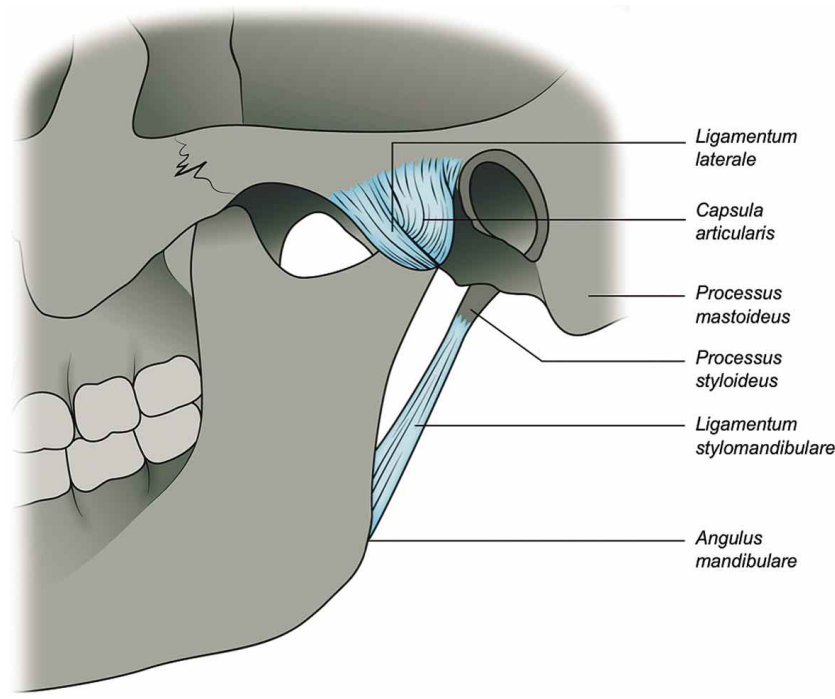

Fig. 1. Esquema de la vista lateral del lado izquierdo del cráneo. Se puede observar que el ligamento estilomandibular (Ligamentum stylomandibulare) se origina en el proceso estiloideo y se inserta en el ángulo mandibular y margen posterior de la rama de la mandíbula, como ha sido descrito en la mayoría de la literatura revisada.

Tabla I. Denominación del ligamento estilomandibular (Ligamentum stylomandibulare) en la literatura científica.

\section{Estilomandibular}

(Término usado por TAI, 2001)

Estilomaxilar

Ligamentum stylomandibulare (Término usado por TAI, 2001)
Giambartolomei (2003); Langton \& Eggleton (1992); Bumann \& Lotzmann (2000); Okeson (2003); Wurgaft \& Montenegro (2003); Maciel (2003); Pertes \& Gross (2005); Rubiano (2005); Sosa (2006); Katchburian \& Arana (2004); Romanes (1968); Velayos \& Santana (2001); Alves \& Candido (2009); MacKinnon \& Morris (1993); Lopes (2004); Latarjet \& Ruiz Liard (2005); Is berg (2003); Sicher \& Dubrul (1991).

Giambartolomei (2003); Ramfjord \& Ash (1972); Martínez Ross (1992); Latarjet \& Ruiz Liard (2005).

Velayos \& Santana (2001). 
FUENTES, R.; OTTONE, N.; CANTIN, M. \& BUCCHI, C. Análisis de los Términos Utilizados en la Literatura Científica para Referirse a los Ligamentos Extracapsulares de la Articulación Témporomandibular Parte II: Ligamentum stylomandibulare y Raphe pterymandibulare. Int. J. Morphol., 32(4):1289-1295, 2014.

Tabla II. Denominación del rafe pterigomandibular (raphe pterymandibulare) en la literatura científica.

\begin{tabular}{ll}
\hline Ligamento pterigomandibular & Giambartolomei (2003); Rubiano; Sosa; Velayos \& Santana (2001); Lopes \\
& (2004); Latarjet \& Ruiz Liard (2005). \\
Ligamento pterigomaxilar & Giambartolomei (2003); Martínez Ross (1992). \\
A poneurosis bucofaringea & Giambartolomei (2003). \\
Ligamentum pterygomandibulare & Velayos \& Santana (2001). \\
Rafe pterigomandibular & Latarjet \& Ruiz Liard (2005) \\
(Término usado por TAI, 2001) & \\
\hline
\end{tabular}

Clasificación del rafe pterigomandibular. Existe controversia con respecto a esta estructura, ya que algunos lo mencionan como ligamento y otros como rafe. Los que lo mencionan como ligamento lo clasifican como uno de los tres ligamentos accesorios (Giambartolomei; Lopes), de acción indirecta (Giambartolomei), auxiliares (Martínez Ross; Rubiano), o extrínsecos (Lopes; Latarjet \& Ruiz) de la ATM. Estos tres ligamentos corresponderían a ligamento esfenomándibular, estilomándibular y rafe pterigomándibular, según esos autores. Ningún autor lo clasifica como un ligamento extracapsular.

Origen del ligamento estilomandibular (Ligamentum stylomandibulare). La mayoría describe el origen de este ligamento, de manera general, en el proceso estiloides (Alves \& Candido; Bumann \& Lotzmann; Maciel; Pertes \& Gross; Katchburian \& Arana; Romanes; Sicher y Dubrul) o en la apófisis estiloides (Okeson; Ramfjord \& Ash; Wurgaft \& Montenegro; Rubiano; Velayos \&
Santana; MacKinnon \& Morris; Isberg) (sic). Otros lo describen en el vértice de la apófisis estiloides (Latarjet \& Ruiz) o cerca de este (Giambartolomei; Martínez Ross) y otros, en la punta (ápex) del estiloides (Langton \& Eggleton) (sic) o encima del proceso estiloides, próximo a su vértice (Lopes) (sic).

Origen del rafe pterigomandibular. Los autores lo describen en el gancho del ala medial (Velayos \& Santana, Giambartolomei) (sic), ala interna (Giambartolomei; Martínez Ross) (sic) o lamina medial (Latarjet \& Ruiz) de la apófisis pterigoides (sic). Rubiano (2005) describe el origen en el gancho del ala externa de la apófisis pterigoides del esfenoides (sic).

Inserción . En la Tabla III Inserción del ligamento estilomandibular (Ligamentum stylomandibulare) se describe la inserción del ligamento estilomandibular, según autor y términos utilizados.

Tabla III. Inserción del ligamento estilomandibular (Ligamentum stylomandibulare), según autor.

\begin{tabular}{|c|c|}
\hline Inserción del Ligamento estilomandibular & Autor \\
\hline Ángulo de la mandíbula & $\begin{array}{l}\text { Wurgaft \& Montenegro (2003); Katchburian \& } \\
\text { Arana (2004); Alves \& Candido (2009); } \\
\text { MacKinnon \& Morris (1993), Sicher \& Dubrul } \\
\text { (1991) }\end{array}$ \\
\hline Ángulo y el borde posterior de la mandíbula (sic). & Isberg (2003) \\
\hline Ángulo y el borde posterior de la rama de la mandíbula (sic). & Okeson (2003) \\
\hline Ángulo del maxilar y el borde posterior de la rama asc endente (sic). & Ramfjord \& Ash (1972) \\
\hline $\begin{array}{l}\text { Borde parotídeo de la mandíbula un poco por encima del ángulo o en el } \\
\text { mismo }\end{array}$ & Giambartolomei (2003) \\
\hline $\begin{array}{l}\text { Margen parotídeo de la mandíbula, alargándose e i nsertándose en la } \\
\text { tuberosidad pterigoidea del ángulo de la mandíbula (sic). }\end{array}$ & Lopes (2004) \\
\hline $\begin{array}{l}\text { Borde posterior medial de la mandíbula entre la inserción del musculo } \\
\text { masetero y del pterigoideo medial }(\text { sic). }\end{array}$ & Langton \& Eggleton (1992) \\
\hline $\begin{array}{l}\text { Borde posterior de la rama de la mandíbula, justamente por encima de su } \\
\text { ángulo, llegando algunas de sus fibras hasta el hioides ( } \mathrm{sic} \text { ). }\end{array}$ & Velayos \& Santana (2001) \\
\hline $\begin{array}{l}\text { El margen posterior del ángulo de la mandíbula y su porción mayor irradia } \\
\text { hasta la fascia del musculo pterigoideo medial (sic). }\end{array}$ & Bumann \& Lotzmann (2000) \\
\hline $\begin{array}{l}\text { Alrededor del ángulo de la mandíbula y de la fascia pterigoidea medial } \\
(\text { sic). }\end{array}$ & Maciel (2003) \\
\hline Superficie medial del borde de la mandibular junto al ángulo (sic). & Pertes \& Gross (2005) \\
\hline $\begin{array}{l}\text { En el tercio inferior del borde posterior de la rama ascendente del maxilar } \\
\text { inferior }(s i c) \text {. }\end{array}$ & Martínez Ross (1992) \\
\hline Parte posterior de la rama montante (sic). & Rubiano (2005) \\
\hline Borde posterior de la rama de la mandíbula (sic). & Romanes (1968); Latarjet \& Ruiz Liard (2005) \\
\hline
\end{tabular}


FUENTES, R.; OTTONE, N.; CANTIN, M. \& BUCCHI, C. Análisis de los Términos Utilizados en la Literatura Científica para Referirse a los Ligamentos Extracapsulares de la Articulación Témporomandibular Parte II: Ligamentum stylomandibulare y Raphe pterymandibulare. Int. J. Morphol., 32(4):1289-1295, 2014.

Inserción del rafe pterigomandibular. La inserción del rafe pterigomandibular se describe en la parte posterior del borde alveolar de la mandíbula (Latarjet \& Ruiz, Giambartolomei) (sic) o reborde alveolar del maxilar inferior (Martínez Ross) (sic). Otros lo describen en la zona retromolar de la mandíbula (Velayos \& Santana) o en la línea oblicua interna, posterior al último molar (Rubiano) (sic).

Función del ligamento estilomandibular (Ligamentum stylomandibulare). Varios autores consideran que este ligamento juega un rol en limitar ciertos movimientos de la dinámica mandibular. Para algunos limita movimientos protrusivos (Wurgaft \& Montenegro; Bumann \& Lotzmann) y mediotrusivos (Bumann \& Lotzmann). Para otros limita sólo la protrusión excesiva de la mandíbula (Okeson; Pertes \& Gross; Sicher y Dubrul). Langton \& Eggleton (1992) describen que asiste a la mandíbula en las fases de traslación de la apertura y cierre y Bumann \& Lotzmann (2000) indican que el ligamento estilomandibular impide la rotación craneal excesiva de la mandíbula. Sin embargo, para varios autores este ligamento no desempeña ningún papel en la dinámica articular, por lo menos en la edad adulta (Lopes) y que no tendría influencia biomecánica significativa (Maciel).

Se describe que este ligamento juega un importante rol anatómico, al ser un preciso marco quirúrgico para la localización, exposición y ligadura de la arteria carótida externa en la fosa retromandibular (Sicher y Dubrul) (sic). Además, podría reforzar la cara medial de la ATM (MacKinnon \& Morris) (sic), por lo que tendría un papel de protección (Velayos \& Santana; Maciel) y estabilidad de esta (Sosa).

Para Lopes (2004) y Latarjet \& Ruiz Liard (2005), el ligamento estilomandibular no es más que un pseudoligamento, siendo un residuo fibroso de un fascículo muscular (sic). Otros lo consideran parte de la fascia cervical (MacKinnon \& Morris; Katchburian \& Arana) o de la fascia profunda del cuello (Bumann \& Lotzmann) (sic).

Función del rafe pterigomandibular. La mayoría de los autores coincide en que esta estructura sirve de punto de inserción común para el músculo buccinador por ventral, y para el músculo constrictor superior de la faringe por dorsal (Martínez Ross; Velayos \& Santana; Giambartolomei; Latarjet \& Ruiz Liard), siendo más bien un puente aponeurótico (Martínez Ross). Según Velayos \& Santana (2001) constituye una referencia importante en la anestesia del nervio alveolar inferior y para Sosa (2006) ayuda a mantener la estabilidad mandibular. Sin embargo, Lopes (2004) describe que no desempeña ningún papel en la dinámica articular, considerándolo un pseudoligamento.

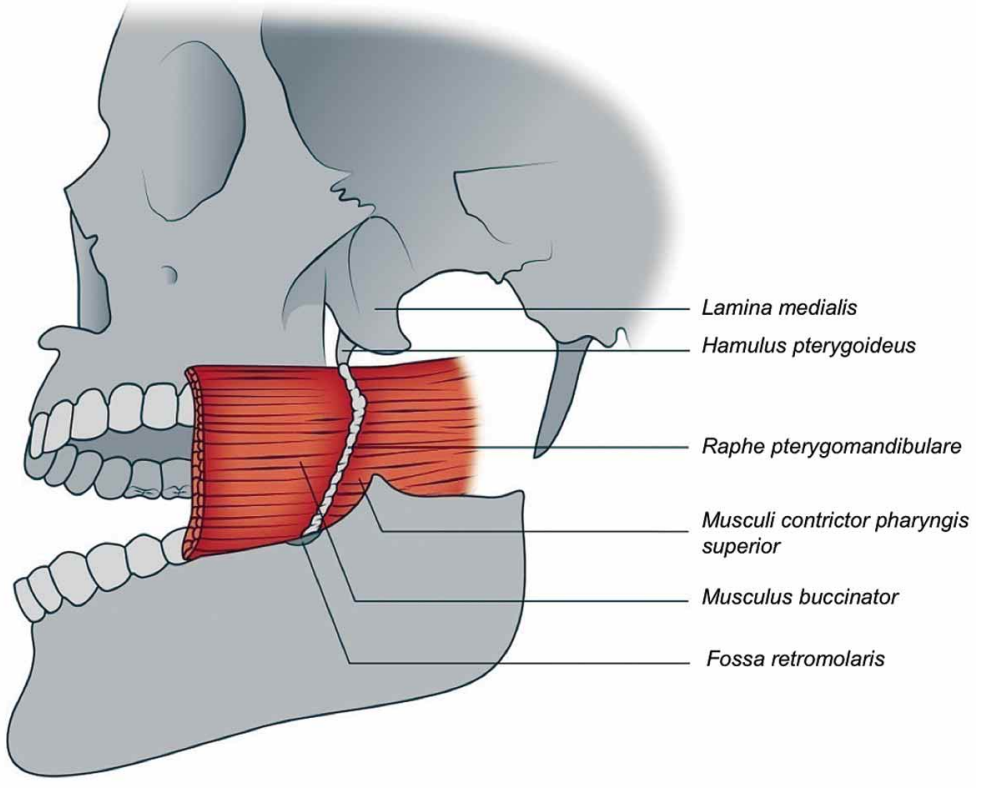

Fig. 2. Esquema de la vista lateral del lado izquierdo del cráneo. Se puede observar que el rafe pterigomandibular (Raphe pterymandibulare) se origina en el gancho de la lamina medial del proceso pterigoideo y se inserta en la fosa retromolar, como ha sido descrito en la mayoría de la literatura revisada. Se esquematizan también la inserción de los músculos buccinador y constrictor superior de la faringe.

\section{DISCUSIÒN}

A fines del siglo XIX, en 1895, nace la Nomina Anatómica de Basilea (Hollinshead, 1983). La Nomina incluía 4 mil quinientos términos aproximadamente, representando una reducción cuantitativa y significativa de los términos anatómicos, que hasta ese entonces eran aproximadamente 50 mil. Esta reducción se debe a la determinación de utilizar un sólo término por estructura anatómica. Tras varias ratificaciones (Duque et al., 2012) la Nomina Anatómica deriva a la actual Terminología Anatómica Internacional (Whitmore, 1999).

En la TAI publicada en 1998, los términos son presentados en tres columnas, y cada término está en latín, inglés y una tercera columna tiene un número de identificación único. En el año 2001 se publica la TAI que incluye el idioma español. El latín fue escogido para la TAI ya que es un idioma muerto, por lo tanto no evoluciona y tampoco pertenece a un país en particular (Whitmore). 
FUENTES, R.; OTTONE, N.; CANTIN, M. \& BUCCHI, C. Análisis de los Términos Utilizados en la Literatura Científica para Referirse a los Ligamentos Extracapsulares de la Articulación Témporomandibular Parte II: Ligamentum stylomandibulare y Raphe pterymandibulare. Int. J. Morphol., 32(4):1289-1295, 2014.

El detalle de los términos usados en la literatura científica y su contraste con la Terminología Anatómica Internacional (2001) se encuentra en las tablas IV y V para el ligamento estilomandibular y rafe pterigomandibular, respectivamente.

\section{Equivalencia terminológica Ligamentum stylomandibulare}

En la Tabla IV se observan los términos usados en la literatura referente al ligamento estilomandibular y estructuras anatómicas asociadas y términos según TAI (2001).

No hay un acuerdo en la literatura revisada sobre cuál es el origen e inserción del ligamento estilomandibular. Sin embargo, lo más descrito es que se origina en el proceso estiloides, cercano al vértice de éste y se inserta en el ángulo y borde posterior de la rama de la mandíbula. Su clasificación correspondería a ligamento extracapsular. Se pueden observar con mayor claridad estas relaciones anatómicas en la Figura 1. En la Tabla V se pueden observar los términos usados en la literatura referente al rafe pterigomandibular y estructuras anatómicas asociadas y términos según TAI (2001). En la Figura 2 se esquematizan las relaciones anatómicas del rafe pterigomandibular, como la describen la mayoría de los autores. En relación al rafe pterigomandibular, según la TAI, esta estructura corresponde efectivamente a un rafe. Sin embargo, la mayoría de los autores lo menciona como un ligamento o pseudoligamento (Martínez Ross; Lopes; Giambartolomei; Rubiano; Sosa; Velayos \& Santana).

Un rafe es una línea de fusión que une dos partes embriológicas simétricas (Antony \& Holden, 2002). La mayoría de los rafes se encuentran en la línea mediana del cuerpo humano, como el rafe palatino (raphe palati), rafe perineal (raphe perinei) y rafe faríngeo (raphe pharyngis), entre otros y no tendrían injerencia en el movimiento de las estructuras relacionadas a él. Por su parte, los ligamentos son estructuras que, por lo general, unen las superficies articulares de dos huesos (Schöttle et al., 2012) y pueden influir en la dinámica articular (Bumann \& Lozmann; Wurgaft \& Montenegro).

El rafe pterigomandibular tiene características descritas de un ligamento y de un rafe, lo que causa confusión con respecto a su denominación. Por un lado, en él se insertan los músculos buccinador y constrictor superior de la faringe (Latarjet \& Ruiz; Martínez Ross; Giambartolomei; Velayos \& Santana) que, si bien no son estructuras simétricas, ocupan en algunos casos toda su extensión (Shimada \& Gasser, 1989). Sin embargo, esta estructura une dos superficies articulares óseas y se inserta en la fosa retromolar (Velayos \& Santana), por lo que participaría además, en la dinámica mandibular (Sosa).

Tabla IV. Términos usados en la literatura referente al ligamento estilomandibular y estructuras anatómicas asociadas y términos según TAI (2001).

$\begin{array}{ll}\begin{array}{l}\text { Terminologia Anatomica Internacional (2001) } \\ \text { (Denominación en latín y español) }\end{array} & \begin{array}{l}\text { Términos usados en los libr os estudiados (ver } \\ \text { referencias en los resultados) }\end{array}\end{array}$

Ligamentum stylomandibulare (Ligamento estilomandibular)

Ligamenta extracapsularia (Ligamentos extracapsulares)

Processus styloideus (Proceso estiloides)

Apex (Vértice)

Angulus mandibulae (Ángulo de la mandíbula)

Margo posterior (Margen posterior)

Ramus mandibulae (Rama de la mandíbula)
Ligamento estilomaxilar

Ligamento estilomandibular

Ligamentum stylomandibulare

Accesorio

Auxiliar

Asociado

De acción indirecta

Extrínseco

Apófisis estiloides

Proceso estiloides

Vértice de la apófisis estiloides

Punta (ápex) de la apófisis estiloides

Ángulo del maxilar

Ángulo de la mandibula

Borde posterior

Borde parotídeo

Margen parotídeo

Margen posterior

Parte posterior de la rama

Rama ascendente

Rama montante

Rama de la Mandíbula 
FUENTES, R.; OTTONE, N.; CANTIN, M. \& BUCCHI, C. Análisis de los Términos Utilizados en la Literatura Científica para Referirse a los Ligamentos Extracapsulares de la Articulación Témporomandibular Parte II: Ligamentum stylomandibulare y Raphe pterymandibulare. Int. J. Morphol., 32(4):1289-1295, 2014.

Tabla V. Términos usados en la literatura referente al rafe pterigomandibular y estructuras anatómicas asociadas y términos según TAI (2001).

\begin{tabular}{|c|c|}
\hline $\begin{array}{l}\text { Terminología Anatómica Internacional (2001) } \\
\text { (Denominación latín y español) }\end{array}$ & $\begin{array}{l}\text { Términos usados en los libr os estudiados (ver } \\
\text { referencias en los resultados) }\end{array}$ \\
\hline Raphe pterymandibulare (Rafe pterigomandibular) & $\begin{array}{l}\text { Ligamento pterigomandibular } \\
\text { Ligamento pterigomaxilar } \\
\text { A poneurosis bucofaríngea. } \\
\text { Ligamentum pterigomandibulare }\end{array}$ \\
\hline Processus pterygoideus (Proceso pterigoides) & Apófisis pterigoides \\
\hline $\begin{array}{l}\text { Hamulus pterygoideus (Gancho del proceso } \\
\text { pterigoides) }\end{array}$ & Gancho \\
\hline Lamina medialis (Lamina medial) & $\begin{array}{l}\text { Ala interna } \\
\text { Ala medial } \\
\text { Lámina medial }\end{array}$ \\
\hline Lamina lateralis (Lamina lateral) & Ala externa \\
\hline Arcus alveolaris (Arco alveolar) & $\begin{array}{l}\text { Borde alveolar } \\
\text { Reborde alveolar }\end{array}$ \\
\hline Fossa retromolaris (Fosa retromolar) & zona retromolar \\
\hline Linea obliqua (Línea oblicua) & Línea oblicua interna \\
\hline Mandibula (Mandíbula) & $\begin{array}{l}\text { Maxilar infe rior } \\
\text { Mandíbula }\end{array}$ \\
\hline
\end{tabular}

En un estudio realizado por Shimada \& Gasser (1989) se describe que en el $36 \%$ de los 60 cadáveres embalsamados adultos caucásicos y negros, existe una completa ausencia del rafe, existiendo una continuidad entre el musculo buccinador y el musculo constrictor superior de la faringe. En otro $36 \%$ se exhibe una fascia amplia que separa los músculos totalmente. En el $28 \%$ restante exhibieron un rafe que pudo ser identificado sólo en su porción superior, y era de forma triangular. Sin embargo, en ese mismo estudio todos los cadáveres de fetos (50) mostraron una amplia fascia que separaba los dos músculos, lo que indica que los cambios en la forma del rafe se producen después del nacimiento. Además, los autores señalan que el rafe en los adultos, con la extensión e inserción descrita en los libros, no fue observada en ninguno de los casos (Shimada \& Gasser, 1989). Esto contrasta con la investigación realizada por Shimada (1979) tras examinar 57 cadáveres japoneses adultos donde se describe que en el $24,7 \%$ de los cadáveres adultos el rafe presenta una amplia forma triangular en su extremo superior, se estrecha hacia inferior y se extiende hasta la mandíbula. En un $9,1 \%$ de los casos se describió al rafe como una banda vertical estrecha que separa completamente los dos músculos.

Se puede observar que no existe claridad en la literatura sobre si la estructura denominada por la TAI como rafe pterigomandibular, es efectivamente un rafe o un ligamento. Esto determina la necesidad de recurrir a los especialistas en TAI, y plantear en el ámbito del SILAT (Simposio Ibero Latinoamericano de Terminología) la confusión existente en referencia a esta estructura para poder establecer su denominación definitiva.

A partir del análisis realizado, queda en evidencia que una cantidad importante de la literatura revisada no menciona las estructuras anatómicas según lo establecido en la TAI. Los libros siguen siendo la principal fuente de estudio de los estudiantes de pregrado, y la falta de unificación, tanto en los términos como en la descripción de las estructuras anatómicas, puede generar confusión y dificultar el proceso de aprendizaje.

FUENTES, R.; OTTONE, N.; CANTIN, M. \& BUCCHI, C. Analysis of terms used in scientific literature referred to extracapsular ligaments of the temporomandibular joint. Part II: Ligamentum stylomandibulare and Raphe pterymandibulare. Int. J. Morphol., 32(4):1289-1295, 2014.

SUMMARY: The Anatomical Terminology (TAI) is the result of centuries of scientific work to unify the terms referring to the anatomical structures of the human body and facilitate communication between the scientific community. However, its use in the literature has not been fully implemented. The objective of this research was to analyze the literature regarding the correct implementation of the terms relating to stylomandibular ligament and pterigomandibular raphe. A review was performed in anatomy text books and scientific articles. Differences in the terms used were found to describe both pterigomandibular ligament and stylomandibular raphe, and also to describe structures that serve as origin and insertion of these, when compared with the terms established by the TAI in Latin, English and Spanish.

KEY WORDS: Stylomandibular Ligament; Pterigomandibular Ligament; Raphe pterigomandibular; Anatomical Terminology. 
FUENTES, R.; OTTONE, N.; CANTIN, M. \& BUCCHI, C. Análisis de los Términos Utilizados en la Literatura Científica para Referirse a los Ligamentos Extracapsulares de la Articulación Témporomandibular Parte II: Ligamentum stylomandibulare y Raphe pterymandibulare. Int. J. Morphol., 32(4):1289-1295, 2014.

\section{REFERENCIAS BIBLIOGRÁFICAS}

Alves, N. \& Candido, P. L. Anatomía Aplicada a la Odontología. São Paulo, Santos, 2009.

Antony, F.C. \& Holden, C.A. Diffuse hypertrichosis and faun-tail naevus as cutaneous markers of spinal dysraphism. Clin. Exp. Dermatol., 27(8):645-8, 2002.

Bumann, A. \& Lotzmann, U. Atlas de Diagnóstico Funcional y Principios Terapeúticos en Odontología. Barcelona, Masson, 2000.

Comité Federal sobre Terminología Anatómica (FCAT). Terminología Anatómica. Terminología Anatómica Internacional. Madrid, Ed. Médica Panamericana, 2001.

Duque, J.; Barco, J. \& Duque, N. Refining the Anatomical Terminology: Opening and Foramen, Two Similarities and Redundancy. Int. J. Morphol., 30(4):1497-1499, 2012.

Federative International Committee on Anatomical Terminologies (FCAT). Terminologia Anatomica. International Anatomical Terminology. New York, Thieme, 1998.

Fuentes, R.; Bucchi, C. \& Cantin, M. A study of terms used in scientific literature to refer to extracapsular ligaments of the temporomandibular joint. Part I: Ligamentum sphenomandibulare. Int. J. Morphol., 32(3):981-986, 2014.

Giambartolomei, L. A. Anatomía del complejo articular cráneomandibular. Villa María, Red de Editoriales de Universidades Nacionales, 2003.

Grau, I.; Fernández, K.; González, G. \& Osorio, M. Algunas consideraciones sobre los trastornos temporomandibulares. Rev. $\mathrm{Cu}$ bana Estomatol., 42(3):0-0, 2005.

Hollinshead, W. H. Anatomía para cirujanos dentistas. México, Harla y Harper \& Row Latinoamericana, 1983.

Isberg, A. Disfunción de la Articulación Temporomandibular. Una Guía Práctica para el Profesional. $2^{\mathrm{a}}$ ed. São Paulo, Artes Médicas Latinoamerica, 2003.

Katchburian, E. \& Arana, V. Histología e Embriología Oral. $2^{\mathrm{a}}$ ed. São Paulo, Médica Panamericana, 2004.

Langton, D. \& Eggleton, T. Functional Anatomy of the Temporomandibular Joint Complex. Tucson, INFORC publications, 1992.

Latarjet, M. \& Ruiz Liard, A. Anatomía Humana. Tomo 2. $2^{\mathrm{a}}$ ed. Buenos Aires, Médica Panamericana, 2005.

Lopes, A. Anatomía Cabeça e Pescoça. Rio de Janeiro, GuanabaraKoogan, 2004.

Maciel, R. N. ATM e Dores Craneofaçiais. Fisiopatología Básica. Sao Paulo, Santos, 2003.
MacKinnon, P. \& Morris, J. Oxford Anatomía Funcional, Cabeza y Cuello, Volumen III. Buenos Aires, Editorial Médica Panamericana, 1993.

Martínez Ross, E. Disfunción Témporomandibular. Santafé, Ediciones Monserrate, 1992.

Okeson, J. Tratamiento de Oclusión y Afecciones Temporomandibulares. 5a ed. Madrid, Mosby Inc., 2003.

Pertes, R. \& Gross, S. Tratamento Clínico das disfunçoes Temporomandibulares e da dor Orofacial. São Paulo, Quintessence, 2005.

Ramfjord, S. \& Ash, M. Oclusión. 2a ed. Filadelfia, Editorial Interamericana, 1972.

Romanes, G. J. Cunningham's Manual of Practical Anatomy: Volume III: Head, Neck and Brain. 15ba Ed. Oxford, Oxford Medical Publications, 1968.

Rubiano, C. M. Tratamiento con Placas y Corrección Oclusal por Tallado Selectivo. Bogotá, Amolca, 2005.

Schöttle, P.B., Hensler, D. \& Imhoff, A.B. Anatomical double-bundle MPFL reconstruction with an aperture fixation. Knee Surg. Sports Traumatol. Arthrosc., 18(2):147-51, 2010.

Shimada, K. Study of the morphology of the raphe pterygomandibularis,Part 1. The adults (in Japanese). Nihon Univ. Dent. J., 53:998-1007, 1979.

Shimada, K. \& Gasser, R. F. Morphology of the pterygomandibular raphe in human fetuses and adults. Anat. Rec., 224(1):11722,1989 .

Sicher, H. \& Dubrul, E. Anatomía oral. 8 Ed. Sao Paulo, Artes Medicas, 1991.

Sosa, G. E. Detección Precoz de los Desórdenes Témporomandibulares. Caracas, Amolca, 2006.

Velayos, J. L. \& Santana H. D. Anatomía de la Cabeza con Enfoque Odontoestomatológico. 3a ed. Madrid, Médica Panamericana, 2001.

Whitmore, I. Terminologia Anatomica: new terminology for the new anatomist. Anat. Rec. 257(2):50-3, 1999.

Wurgaft, R. \& Montenegro, M. A. Desarrollo y Estructura de la Articulación Temporomandibular. Santiago, Servimpres, 2003.

Dirección para Correspondencia:

Dr. Ramón Fuentes Fernández

Facultad de Odontología

Universidad de La Frontera

Manuel Montt 112

Recibido : 05-05-2014

Casilla 54-D, Temuco

Aceptado: 22-08-2014

CHILE

Email: ramon.fuentes@ufrontera.cl 